\title{
Growth of InAsN/InGaAs(P) quantum wells on InP by gas source molecular beam epitaxy
}

\author{
Jyh-Shyang Wang, ${ }^{a}$ Hao-Hsiung Lin, Li-Wei Song, and Guan-Ru Chen \\ Department of Electrical Engineering, Room 419, National Taiwan University, Taipei, \\ Taiwan, Republic of China
}

(Received 10 November 1999; accepted 9 October 2000)

\begin{abstract}
The growth of InAsN/InGaAs(P) quantum wells (QWs) on InP substrates by gas-source molecular-beam epitaxy and a rf plasma nitrogen source is reported for the first time. The double crystal $x$-ray diffraction satellite peaks of the InAsN/InGaAsP multiple quantum well (MQW) samples are sharper than those of the pure InAs/InGaAsP MQW samples, showing that a flatter heterointerface is achieved due to the smaller lattice mismatch. However, broadening of the satellite peaks and degradation of the photoluminescence (PL) intensity due to the increase of the nitrogen composition in these InAsN/InGaAsP MQWs suggest the existence of defects introduced by the small diameter nitrogen atoms located on arsenic sites. The PL result also shows that the peak energy decreases as the nitrogen composition increases. The estimated transition energy shrinkage coefficient is $-31 \mathrm{meV} / \mathrm{at}$ \% nitrogen. The largest nitrogen composition obtained in this study is $5.9 \%$, and its $10 \mathrm{~K} \mathrm{PL}$ peak wavelength is $\sim 2.6 \mu \mathrm{m}(480 \mathrm{meV})$. The effects of growth temperature on nitrogen composition and PL intensity are also discussed. (c) 2001 American Vacuum Society.
\end{abstract}

[DOI: 10.1116/1.1330265]

\section{INTRODUCTION}

Low-nitrogen-content zinc blende III-V alloys have received much attention in the past few years. ${ }^{1-3}$ The large difference in atomic size and electronegativity between $\mathrm{N}$ and As has motivated a theoretical approach to understand the huge bowing parameter, and also to ascertain the semiconductor or semimetal nature of these alloys. ${ }^{4-6}$ In addition, the large conduction band offset of these materials is very promising for overcoming the poor temperature characteristics of conventional InGaAsP/InP long-wavelength laser diodes. ${ }^{2,3}$

In midinfrared 2-5 $\mu \mathrm{m}$ wavelengths, the InAsN alloy could also be a very promising material. Recently, we demonstrated a high-quality $2.2 \mu \mathrm{m}$ InAs/InGaAs/InP highly strained multiple quantum well (MQW) laser grown by gassource molecular-beam epitaxy. ${ }^{7}$ Using InAsN to replace InAs can ease the critical thickness limitation on the quantum well because of its small lattice constant, and it can also further reduce the band gap energy of the quantum well because of its huge bowing effect. These two features reveal the possibility of pushing the wavelength of lasers on InP substrates to a longer infrared range. For device application, it is very important to improve the material quality; however, the epitaxial growth technique and material properties of In(Ga)AsN have not yet been fully studied. III-V (N) materials may have epitaxial growth problems due to their miscibility gap, which originates from the large differences in atomic radius and electronic negativity. ${ }^{8,9}$ Thus, suitable nitride source deployment and growth conditions are very important for the growth of these materials. A number of epitaxial methods have been used to grow $\operatorname{In}(\mathrm{Ga})$ AsN, including metalorganic chemical vapor deposition (MOCVD), ${ }^{10-12}$

${ }^{a}$ Electronic mail: jswang@mimi.ee.ntu.edu.tw metalorganic molecular-beam epitaxy (MOMBE), ${ }^{13}$ chemical-beam epitaxy $(\mathrm{CBE}),{ }^{14}$ and gas-source molecularbeam epitaxy (GSMBE) ${ }^{15,16}$ In this article we report on GSMBE growth of InAsN/InGaAs(P) QWs on InP substrates, and on their crystal quality and optical properties using double crystal x-ray diffraction (DXRD) and photoluminescence (PL) measurements, respectively.

\section{EXPERIMENT}

The samples were grown on semi-insulating (100) InP substrates using a VG V-80H GSMBE system. Besides the elemental In and Ga sources and thermally cracked $\mathrm{AsH}_{3}$ and $\mathrm{PH}_{3}$ sources used for producing group III and $\mathrm{V}$ molecular beams, an EPI UNI-bulb rf plasma source was used to generate active $\mathrm{N}$ species. The $\mathrm{rf}$ power for these growths was kept at $120 \mathrm{~W}$. For the growth of $\operatorname{InAs}(\mathrm{N}) / \mathrm{InGaAsP} \mathrm{MQW}$ samples, after thermally cleaning the InP substrate at $500{ }^{\circ} \mathrm{C}$ under $\mathrm{P}_{2}$ flux, a 0.5 - $\mu$ m-thick $\mathrm{In}_{0.75} \mathrm{Ga}_{0.25} \mathrm{As}_{0.53} \mathrm{P}_{0.47}$ layer which is lattice matched to the InP substrate was first grown as a buffer layer at a growth rate of $2 \mu \mathrm{m} / \mathrm{h}$. High-brightness mode $\mathrm{N}_{2}$ plasma was then ignited for subsequent $\mathrm{QW}$ growth. The MQW structure is comprised of 20- or 30-nmthick $\mathrm{In}_{0.75} \mathrm{Ga}_{0.25} \mathrm{As}_{0.53} \mathrm{P}_{0.47}$ barriers and 3-nm-thick $\operatorname{InAs}(\mathrm{N})$ wells. The growth rate and As/In flux ratio of the $\operatorname{InAs}(\mathrm{N})$ well were $1.5 \mu \mathrm{m} / \mathrm{h}$ and 2 , respectively. To study the growth conditions, the range of growth temperatures was varied from 340 to $460{ }^{\circ} \mathrm{C}$. And the range of nitrogen flow rates was varied from 0.2 to $1.4 \mathrm{sccm}$, the corresponding beam equivalent pressure (BEP) of which was from 1 to $6 \times 10^{-5}$ mbar. To control illumination of nitrogen species, a mechanical shutter in front of the plasma source was used. However, there were no growth interruptions at the heterointerfaces of the MQW structures. The rf power was turned off immediately after the QW growth was finished. Finally, a $0.1-\mu \mathrm{m}-$ 
TABLE I. Summary of the growth conditions and nitrogen composition of $\operatorname{InAs}_{1-x} \mathrm{~N}_{x} / \operatorname{InGaAs}(\mathrm{P})$ QWs.

\begin{tabular}{ccccc}
\hline \hline $\begin{array}{c}\text { Sample } \\
\text { No. }\end{array}$ & $\begin{array}{c}\text { Number of } \\
\text { periods }\end{array}$ & $\begin{array}{c}\text { Flow rate of } \mathrm{N}_{2} \\
(\mathrm{sccm})\end{array}$ & $\begin{array}{c}\text { Growth temperature } \\
\left({ }^{\circ} \mathrm{C}\right)\end{array}$ & $\begin{array}{c}\text { Nitrogen } \\
\text { composition }\end{array}$ \\
\hline C821 & 10 & 0 & 400 & 0 \\
C822 & 10 & 0.35 & 400 & 0.011 \\
C823 & 10 & 0.70 & 400 & 0.035 \\
C824 & 10 & 1.40 & 400 & 0.059 \\
C827 & 4 & 0.43 & 430 & 0.022 \\
C828 & 4 & 0.43 & 400 & 0.025 \\
C829 & 4 & 0.43 & 370 & 0.027 \\
C830 & 4 & 0.43 & 340 & 0.033 \\
C885 & 1 & 0.43 & 420 & $\cdots$ \\
C886 & 1 & 0.43 & 440 & $\cdots$ \\
C887 & 1 & 0.43 & 460 & $\ldots$ \\
C888 & 1 & 0.43 & 420 & $\cdots$ \\
C889 & 1 & 0.43 & 400 & $\cdots$ \\
\hline \hline
\end{tabular}

thick InP cap layer was overgrown on the QW. Detailed growth conditions are summarized in Table I. The typical flow rate fluctuation was within $0.1 \mathrm{sccm}$. The growth of InAs(N)/InGaAs single quantum well (SQW) samples is similar to $\operatorname{InAs}(\mathrm{N}) / \mathrm{InGaAsP} \mathrm{MQW}$ ones. The buffer layer and the barrier are $\mathrm{In}_{0.53} \mathrm{Ga}_{0.47} \mathrm{As}$, and the growth rate was $2.83 \mu \mathrm{m} / \mathrm{h}$.

The nitrogen compositions of InAsN MQWs were determined from the DXRD spectra fittings using commercial dynamic simulation software RADS. The optical quality was evaluated using PL measurements. The $488 \mathrm{~nm}$ line of an argon laser was used as the excitation source and a closed cycle helium cryostat was used for low temperature measurements. The signal was detected using a liquid nitrogen cooled InSb photodiode with a Hamamatsu P3357-02 preamplifier.

\section{RESULTS AND DISCUSSIONS}

Figure 1 shows the DXRD spectra of 10-period $\operatorname{InAs}(\mathrm{N}) /$ InGaAsP MQW structures grown at different nitrogen flow rates. The well and barrier widths are 3 and $20 \mathrm{~nm}$, respectively. Basically, increasing the nitrogen flow rate shifts the envelope and the satellite peaks toward the substrate peak, which indicates the reduction of lattice mismatch due to the introduction of nitrogen. The full width at half maximum (FWHM) of the $m=-3$ satellite peaks is also shown in Fig. 1. Note that the satellite peaks of InAsN/InGaAsP MQW samples $(\mathrm{C} 822, \mathrm{C} 823$, and C824) are sharper than those of the InAsN/InGaAsP MQW sample (C821). This implies that flatter heterointerfaces are achieved in InAsN/InGaAsP samples due to their smaller lattice mismatch. However, the satellite peaks of the InAsN MQW samples broaden in spite of the decreasing lattice mismatch as the nitrogen flow rate increases. The degradation on the crystal quality might be due to the defects or dislocations introduced by the smalldiameter nitrogen atoms located on arsenic sites in InAsN. To analyze the nitrogen composition in InAsN quantum wells, the composition of the InGaAsP buffer and barrier layers was first determined from the peak marked B in Fig. 1.

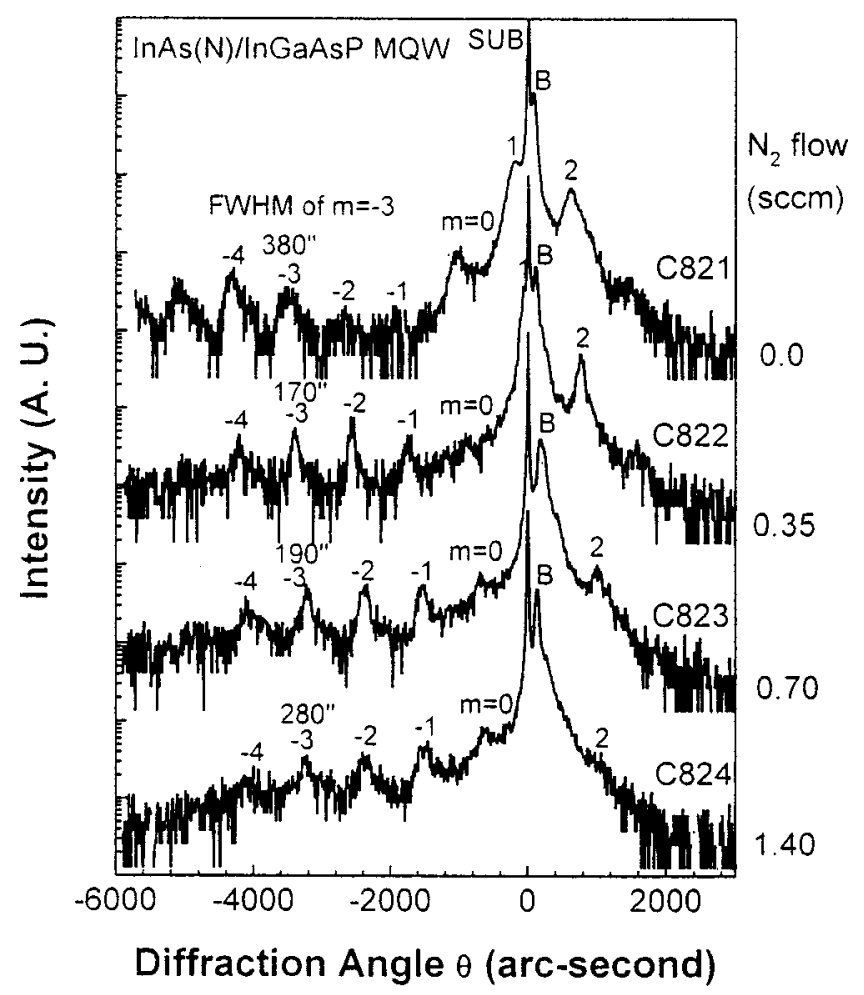

FIG. 1. DXRD spectra of 10-period InAsN/InGaAsP MQWs grown at different nitrogen flow rates. SUB and B denote the peaks of the InP substrate and InGaAsP buffer layer, respectively. The superlattice orders of each satellite peak are marked. The FWHMs of $m=3$ satellite peaks are also shown.

The nitrogen composition was then used as a parameter to fit the DXRD curves using the RAD commercial dynamic simulation software. The typical eror of the nitrogen composition is within 0.002 . The nitrogen composition determined from the DXRD spectra as a function of nitrogen flow rates is plotted in Fig. 2. As can be seen, the incorporation behavior is linear.

The $10 \mathrm{~K}$ PL spectra of the as-grown InAsN/InGaAsP MQWs are shown in Fig. 3(a). As can be seen, the PL peak energy decreases with increasing nitrogen composition. This result indeed confirms the bowing effect due to the incorporation of nitrogen. However, the integrated PL intensity degrades almost linearly with increasing nitrogen composition, as shown in Fig. 3(b), and the FWHM also shows similar behavior. The luminescence of sample C824, whose nitrogen composition is as large as $5.9 \%$, is not even observable. In general, the more $\mathrm{N}$ atoms incorporated into InAs, the poorer the sample crystallinity and the weaker the PL intensity. The degradation is due to residual strain resulting from the small diameter nitrogen atoms located on arsenic sites. And the behavior is similar to that reported previously for the InGaAsN system. ${ }^{1-3,11,12,15,16}$

However, by annealing the samples, the PL intensities can be recovered by decreasing the number of defects. Figure 4 shows the $10 \mathrm{~K}$ PL spectra of the annealed $\operatorname{InAs}(\mathrm{N}) / \mathrm{InGaAsP}$ MQWs. The samples were annealed at $525^{\circ} \mathrm{C}$ for $15 \mathrm{~min}$ in nitrogen atmosphere. To protect the surfaces of the samples during the annealing process, the tops of $\mathrm{Si}$ wafers were 


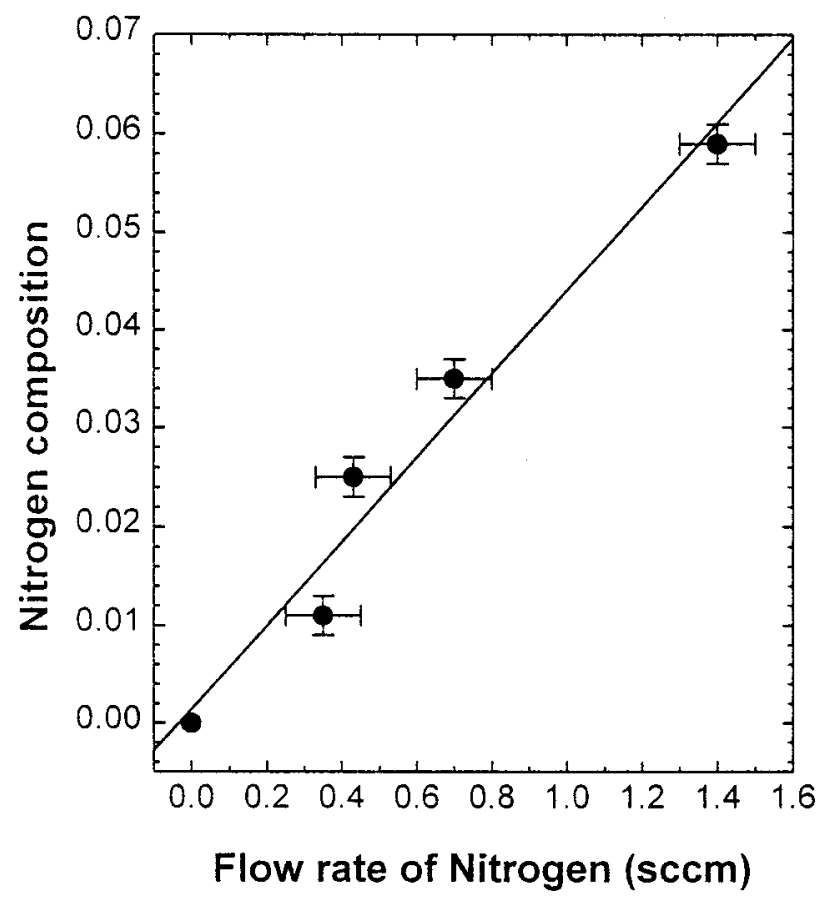

FIG. 2. Nitrogen composition determined from DXRD spectra as a function of nitrogen flow rate.

capped. By comparing the peak energies of the samples before and after annealing, seen in Figs. 3(a) and 4, respectively, almost no structural change or quantum-well intermixing (QWI) is observed under this condition. ${ }^{17}$ Figure 4 also shows the $10 \mathrm{~K} \mathrm{PL}$ spectrum of sample C824, whose luminescence before annealing is not detectable. The nitrogen composition of sample C824 is as high as 5.9\% and its PL peak wavelength is as long as $2.6 \mu \mathrm{m}(480 \mathrm{meV})$. A detailed comparison between as-grown and after annealing samples is summarized in Table II. In both Figs. 2 and 4, the InAs MQW sample, C821, which has the largest compressive strain, shows a low energy shoulder. This shoulder becomes increasingly weaker when the temperature increases and it eventually disappears at $80 \mathrm{~K}$. Because of this behavior, the shoulder is believed to be an impurity or defectrelated luminescence. ${ }^{18,19}$

The $10 \mathrm{~K}$ PL peak energy as a function of nitrogen composition for the annealed InAsN/InGaAsP MQWs is shown in Fig. 5. By linear fitting, the transition energy shrinkage coefficient is $-31 \mathrm{meV} / \mathrm{at}$. \% $\mathrm{N}$. This result, although from MQWs grown on InP, is consistent with the result from the absorption measurements on bulk $\operatorname{InAs}_{1-x} \mathrm{~N}_{x}$ grown on GaAs. ${ }^{10}$ However, it is much smaller than the value in the PL study of the $\mathrm{In}_{0.3} \mathrm{Ga}_{0.7} \mathrm{As}_{1-x} \mathrm{~N}_{x}$ quantum well, $-150 \mathrm{meV} /$ at. \%. N, reported by Kondow et al. ${ }^{2}$

The incorporation behavior of nitrogen in InAsN as a function of growth temperature is shown in Fig. 6. The growth rate, As/In flux ratio, and nitrogen flux for these InAsN layers were fixed at $1.5 \mu \mathrm{m} / \mathrm{h}, 2$, and $0.43 \mathrm{sccm}$, respectively. Detailed growth conditions are summarized in Table I. As can be seen in Fig. 6, the nitrogen composition increases from 0.022 to 0.033 in mole fraction as the growth
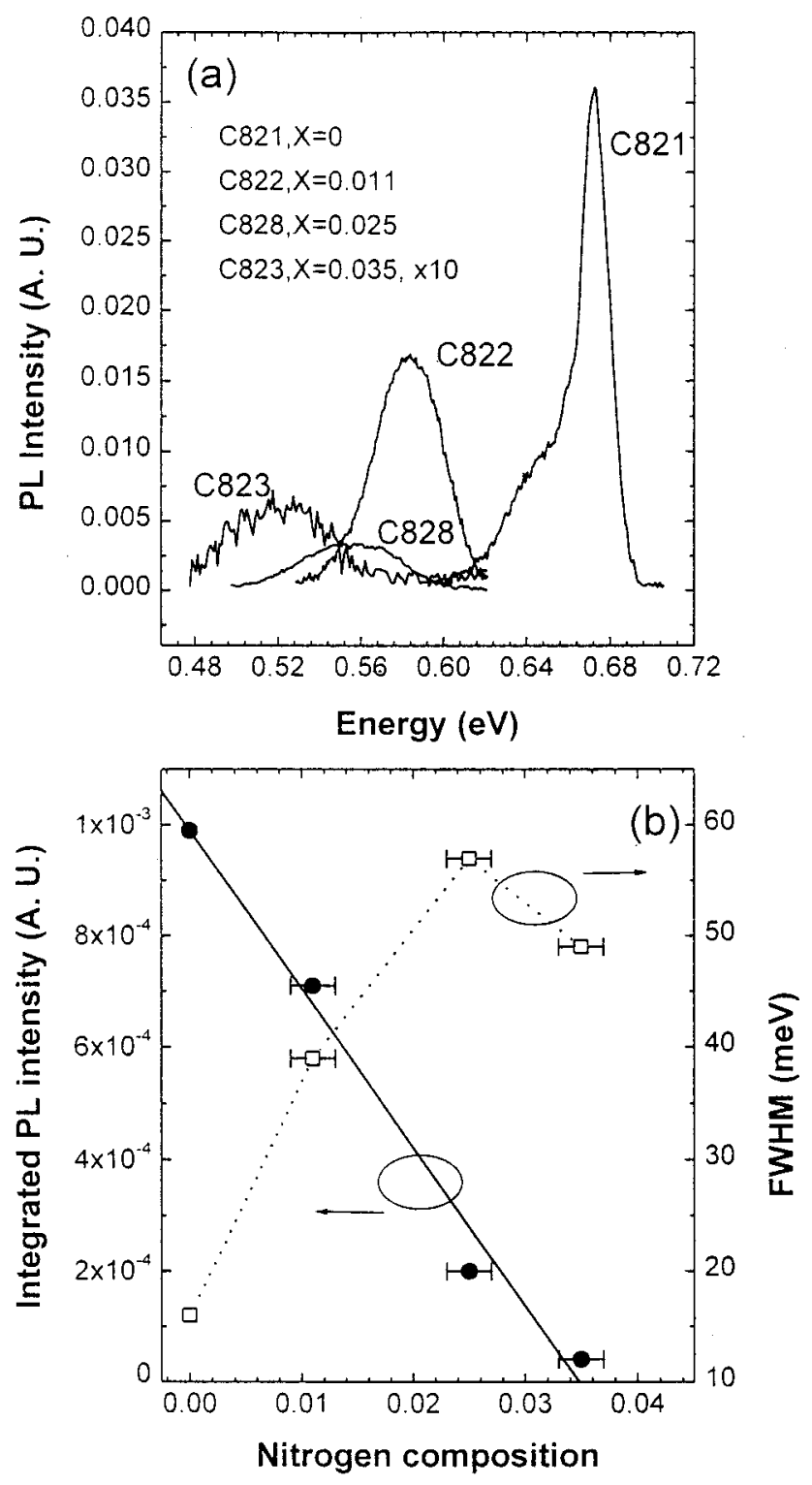

Fig. 3. (a) $10 \mathrm{~K}$ PL spectra of as-grown InAsN/InGaAsP MQWs. (b) Integrated PL intensity and FWHM as a function of nitrogen composition.

temperature decreases from 430 to $340{ }^{\circ} \mathrm{C}$. This is due to the decrease of group $\mathrm{V}$ equilibrium pressure as the growth temperature decreases. The lower re-evaporation rate at lower growth temperature enhances the incorporation of nitrogen. The integrated $10 \mathrm{~K} \mathrm{PL}$ intensity as a function of inverse growth temperature is shown in Fig. 7. Besides the results of the InAsN/InGaAsP MQW samples, the results of InAsN/ InGaAs SQW samples are also plotted in Fig. 7. The growth conditions for the InAsN well are the same, except for the growth temperature, as can be seen in Table I. As shown in Fig. 7, the results of InAsN/InGaAs SQW samples, whose temperature range is higher, display a two-section curve. On the other hand, the InAsN/InGaAsP MQW samples grown at the lower temperature range display a straight line parallel to the low-temperature section of InAsN/InGaAs SQW samples. In general, the formation of the nonradiative defects in these InAsN/InGaAs(P) QWs is not only due to the re- 


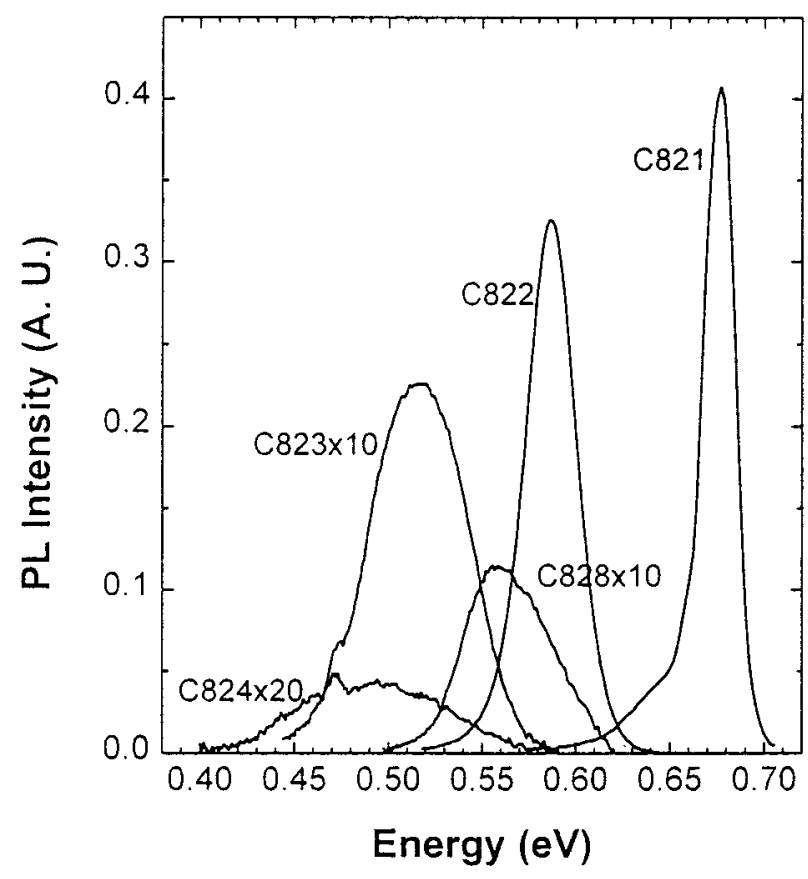

FIG. 4. $10 \mathrm{~K}$ PL spectra of the annealed InAsN/InGaAsP MQWs. These samples were anealed at $525^{\circ} \mathrm{C}$ for $15 \mathrm{~min}$ in nitrogen atmosphere.

sidual strain introduced by the small diameter nitrogen atoms on As sites, but is also related to some native point defects such as vacancies and arsenic antisites induced by lower growth temperature. Novák et al. ${ }^{20}$ reported that the boundary temperature between nonstoichiometric growth and conventional growth for MBE GaAs layers is around $400{ }^{\circ} \mathrm{C}$. In nonstoichiometric growth, there are many native point defects such as vacancies and arsenic antisites induced by lower growth temperature. Although this boundary temperature can vary, depending on the materials and growth conditions, we believe the curves with larger slope at the lower growth temperature region in Fig. 7 are due to native point defects such as vacancies and arsenic antisites. On the other hand, the curve with the smaller slope at the higher growthtemperature region in Fig. 7 is due to the effect of nitrogen composition and is similar to that shown in Fig. 3(b).

TABLE II. Summary of the detailed PL data comparison between as-grown and after annealing samples.

\begin{tabular}{cccccccc}
\hline \hline & \multicolumn{3}{c}{ As grown } & & \multicolumn{3}{c}{ After annealing } \\
\cline { 2 - 3 } \cline { 6 - 8 } $\begin{array}{c}\text { Sample } \\
\text { No. }\end{array}$ & $\begin{array}{c}\text { Peak } \\
\text { energy } \\
(\mathrm{eV})\end{array}$ & $\begin{array}{c}\text { Integrated } \\
\text { intensity } \\
\left(10^{-3}\right)\end{array}$ & $\begin{array}{c}\text { FWHM } \\
(\mathrm{meV})\end{array}$ & $\begin{array}{c}\text { Peak } \\
\text { energy } \\
(\mathrm{eV})\end{array}$ & $\begin{array}{c}\text { Integrated } \\
\text { intensity } \\
\left(10^{-3}\right)\end{array}$ & $\begin{array}{c}\text { FWHM } \\
(\mathrm{meV})\end{array}$ \\
\hline C821 & 0.672 & 1.00 & 16 & & 0.677 & 10.0 & 20 \\
C822 & 0.583 & 0.70 & 39 & & 0.587 & 12.0 & 32 \\
C828 & 0.557 & 0.20 & 57 & & 0.558 & 0.7 & 54 \\
C823 & 0.517 & 0.04 & 49 & & 0.519 & 1.5 & 62 \\
C824 & $\cdots$ & $\cdots$ & $\cdots$ & & 0.480 & 0.2 & 85 \\
\hline \hline
\end{tabular}

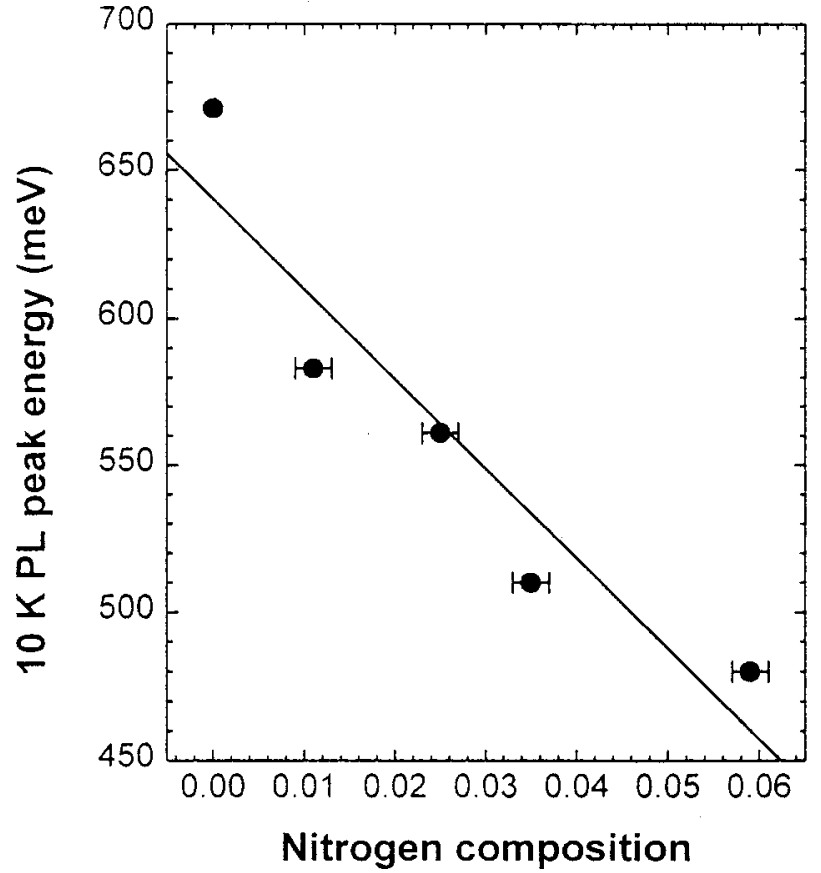

FIG. 5. $10 \mathrm{~K}$ PL peak energies as a function of nitrogen composition $x$ for the annealed InAsN/InGaAsP MQWs. By linear fitting, the estimated transition energy shrinkage coefficient is $-31 \mathrm{meV} / \mathrm{at}$. $\% \mathrm{~N}$.

\section{CONCLUSION}

In summary, the growth of InAsN/InGaAs(P) QWs on an InP substrate with different nitrogen compositions by GSMBE using a rf plasma nitrogen source was investigated for the first time. The DXRD spectra show that a flatter heterointerface is achieved due to the smaller lattice mismatch.

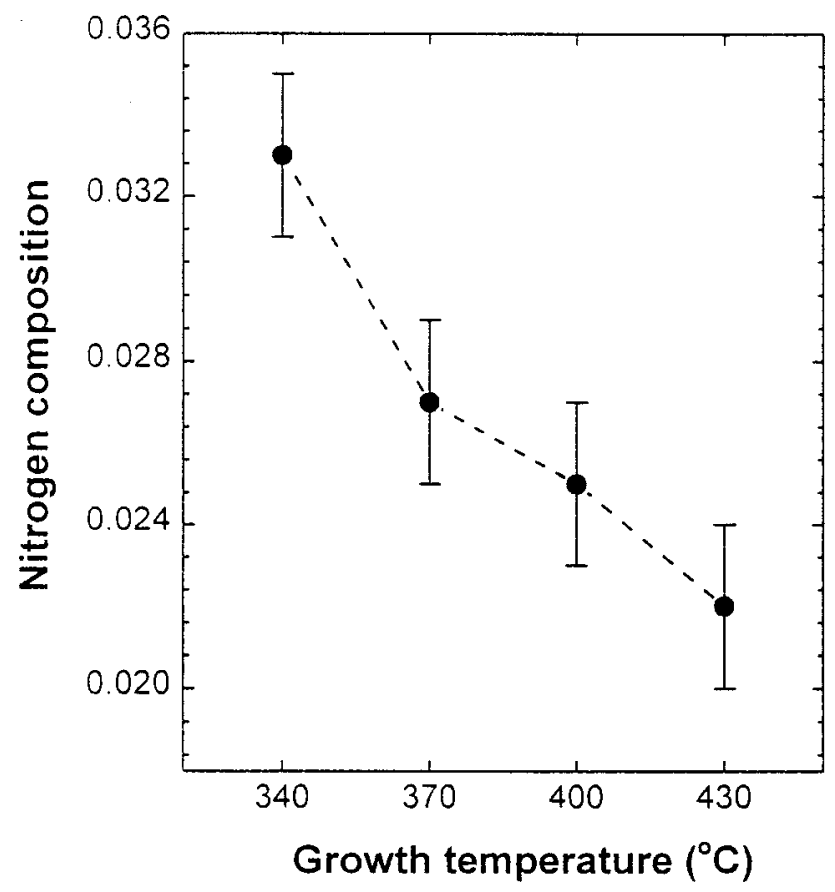

FIG. 6. Nitrogen composition in an InAsN quantum well as a function of growth temperature. 


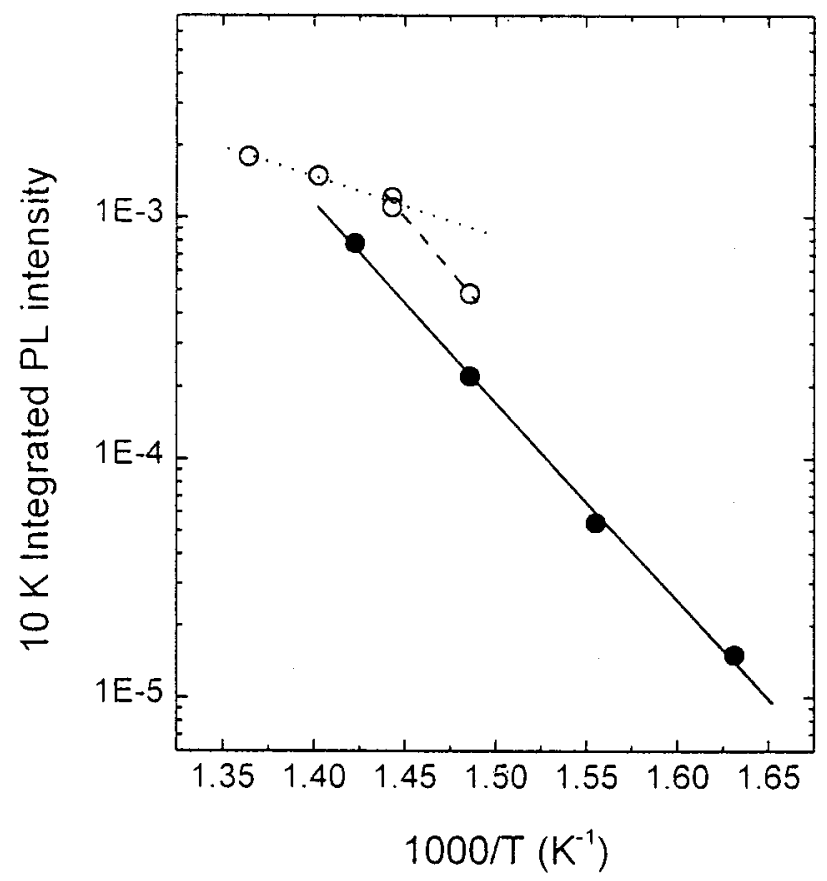

FIG. 7. $10 \mathrm{~K}$ integrated PL intensity as a function of inverse growth temperature. The closed circle and open circle represent InAsN/InGaAsP MQWs and InAsN/InGaAs SQWs, respectively.

However, the broadening of the satellite peaks and the degradation of PL intensity due to the increase of the nitrogen composition in these InAsN MQWs suggest the existence of defects or dislocations introduced by the small-diameter nitrogen atoms located on arsenic sites. The PL result also shows that the peak energy decreases with increasing nitrogen composition in the InAsN layer. A $1 \%$ nitrogen increase in alloy composition causes a shrinkage of $31 \mathrm{meV}$ on the transition energy of the QW. The largest nitrogen composition obtained in this study is $5.9 \%$, and its $10 \mathrm{~K} \mathrm{PL}$ peak wavelength is $\sim 2.6 \mu \mathrm{m}(480 \mathrm{meV})$. The decrease in growth temperature increases nitrogen incorporation. However, the effect of growth temperature on the integrated PL intensity was also studied. In the low-temperature region, the forma- tion of nonradiative defects in these InAsN QWs is not only due to residual strain introduced by the small-diameter nitrogen atoms on As sites, but also, from some native point defects such as as vacancies and arsenic antisites induced by lower growth temperature. However, in the highertemperature region, the slight variation of the PL intensity is due to the change in nitrogen composition.

\section{ACKNOWLEDGMENT}

This work was supported by the National Science Council of the Republic of China under Contract No. NSC 87-2215E-002-010.

${ }^{1}$ M. Kondow, K. Uomi, T. Kitatani, S. Watahiki, and Y. Yazawa, J. Cryst. Growth 164, 175 (1996).

${ }^{2}$ M. Kondow, T. Kitatani, S. Nakatsuka, M. C. Larson, K. Nakahara, Y. Yazawa, M. Okai, and K. Uomi, IEEE J. Sel. Top. Quantum Electron. 3, 719 (1997).

${ }^{3}$ W. G. Bi and C. W. Tu, Appl. Phys. Lett. 72, 1161 (1998).

${ }^{4}$ S. Sakai, Y. Ueta, and Y. Teauchi, Jpn. J. Appl. Phys., Part 1 32, 4413 (1993).

${ }^{5}$ S. H. Wei and A. Zunger, Phys. Rev. Lett. 76, 664 (1996).

${ }^{6}$ L. Bellaiche, S. H. Wei, and A. Zunger, Phys. Rev. B 54, 17568 (1996).

${ }^{7}$ J. S. Wang, H. H. Lin, and L. W. Sung, IEEE J. Quantum Electron. 34, 1959 (1998).

${ }^{8}$ J. Neugebauer and C. G. van de Walle, Phys. Rev. B 51, 10568 (1995).

${ }^{9}$ D. Schlenker, T. Miyamoto, Z. Pan, F. Koyama, and K. Iga, J. Cryst. Growth 196, 67 (1999).

${ }^{10}$ H. Naoi, Y. Naoi, and S. Sakai, Solid-State Electron. 41, 319 (1997).

${ }^{11}$ S. Sato, Y. Osawa, and T. Saitoh, Jpn. J. Appl. Phys., Part 1 36, 2671 (1997).

${ }^{12}$ H. Saito, T. Makimoto, and N. Kobayashi, J. Cryst. Growth 195, 416 (1998).

${ }^{13}$ C. Jin, Y. Qiu, S. A. Nikishin, and H. Temkin, Appl. Phys. Lett. 74, 3516 (1999).

${ }^{14}$ T. Miyamoto, K. Takeuchi, T. Kageyama, F. Koyama, and K. Iga, J. Cryst. Growth 197, 67 (1996).

${ }^{15}$ M. Kondow, T. Kitatani, M. C. Larson, K. Nakahara, K. Uomi, and H. Inoue, J. Cryst. Growth 188, 255 (1998).

${ }^{16}$ H. P. Xin and C. W. Tu, Appl. Phys. Lett. 72, 2442 (1998).

${ }^{17}$ J. S. Wang and H. H. Lin, J. Vac. Sci. Technol. B 17, 1997 (1999).

${ }^{18}$ S. T. Davey, E. G. Scott, B. Wakefield, and G. J. Davies, Semicond. Sci. Technol. 3, 365 (1988).

${ }^{19}$ E. Tournié and K. H. Ploog, Appl. Phys. Lett. 61, 2808 (1992).

${ }^{20}$ J. Novák, M. Kuera, M. Morvic, J. Betko, A. Förster, and P. Kordoš, Mater. Sci. Eng., B 44, 341 (1997). 